\title{
IMPORTANCE OF THE SPIN-ORBIT COUPLING FOR $3 d$-ION COMPOUNDS: THE CASE OF NiO
}

\author{
R.J. RADWAŃSKI ${ }^{a, b}$ AND Z. ROPKA ${ }^{a}$ \\ ${ }^{a}$ Center for Solid State Physics, św. Filip 5, 31-150 Kraków, Poland \\ ${ }^{b}$ Institute of Physics, Pedagogical University \\ Podchorążych 2, 30-084 Kraków, Poland
}

\begin{abstract}
The orbital and spin moment of the $\mathrm{Ni}^{2+}$ ion in $\mathrm{NiO}$ has been calculated at $0 \mathrm{~K}$ to be $0.54 \mu_{\mathrm{B}}$ and $1.99 \mu_{\mathrm{B}}$ respectively. Such large orbital moment, more than $20 \%$ of the total moment of $2.53 \mu_{\mathrm{B}}$, proves the need for the "unquenching" of the orbital moment in compounds containing $3 d$ ions. It turns out that the spin-orbit coupling is indispensable for description of magnetic and electronic properties of $3 d$-ion compounds.
\end{abstract}

PACS numbers: $75.20 . \mathrm{Hr}, 71.70 . \mathrm{Ej}$

The discovery of the high-temperature superconductivity in the copper oxide has revealed the enormous shortage of our general understanding of the $3 d$-ion compounds. Namely, the insulating state of $\mathrm{La}_{2} \mathrm{CuO}_{4}$ contradicts the standard band-structure result that predicts it to be metal. This dramatic breakdown of the ordinary band-electron theory has been known already for years for $3 \mathrm{~d}$-ion monooxides [1-5]. Different reparations do not lead to the consistent picture for $3 d$-ion systems known as the Mott insulators. At present after announcement of the essential importance of the single-ion effects and many-electron discrete states in description of compounds containing transition-metal atoms of the $3 d$ and $4 f$ groups the fundamental controversy has become the scientific fact $[6,7]$. In Ref. [6] we have argued that the spin-orbit ( $\mathrm{s}-\mathrm{o}$ ) coupling is essentially important (indispensable) for the description of electronic and magnetic properties of compounds containing $3 d$ ions. The $s-o$ coupling is largely ignored in the nowadays in-fashion solid-state theories owing to the general conviction about the quenching of the orbital moment and the weakness of the s-o coupling [1-5] (clearly admitted in Ref. [3], p. 7164). Nowadays ionic systems like $\mathrm{LaMnO}_{3}, \mathrm{LaCoO}_{3}, \mathrm{MgV}_{2} \mathrm{O}_{4}$, and $\mathrm{NiO}$ are in fashion. Here we will concentrate on properties of $\mathrm{NiO}$.

The aim of the present short paper is to report results of calculations of the orbital and spin moment. In $\mathrm{NiO}$ the $\mathrm{Ni}$ ions are divalent. Their 6 electrons form the highly-correlated electron system with $S=1$ and $L=3$. The ${ }^{3} F$ term is 21 -fold degenerated $[7,8]$. The $\mathrm{Ni}^{2+}$ ion experiences the crystal field, the spinorbit coupling and the intersite spin-dependent interactions. The latter term is approximated by the mean-field approach. The fine electronic structure resulting 


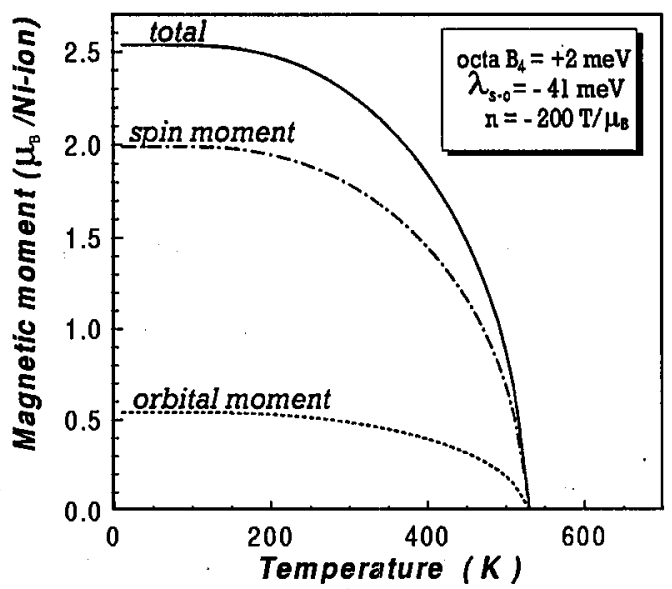

Fig. 1. The temperature dependence of the $\mathrm{Ni}^{2+}$-ion moment in $\mathrm{NiO}$ split into the spin and orbital contribution.

from the cubic crystal-field interactions in the presence of the spin-orbit coupling, presented in Ref. [7], has the triply degenerated crystal-field ground state with the moments of 0 and $\pm 2.14 \mu_{\mathrm{B}}$.

We have calculated (the computer program is available on the written request to the authors) the spin and orbital part of the magnetic moment as well as their temperature dependence. The calculations have been performed by the self-consistent way in order to get the magnetic state with Néel temperature of $525 \mathrm{~K}$. These calculations resemble much the calculations often perfomed by us for rare-earth compounds [9]. The orbital and spin moment of the $\mathrm{Ni}^{2+}$ ion in $\mathrm{NiO}$ has been calculated at $0 \mathrm{~K}$ to be $0.54 \mu_{\mathrm{B}}$ and $1.99 \mu_{\mathrm{B}}$ respectively. For calculations we have taken the spin-orbit coupling constant $\lambda_{\mathrm{s}-\mathrm{o}}=-41 \mathrm{meV}$ and the octahedral crystal-field parameter $B_{4}=+2 \mathrm{meV}$ (it yields the overall crystalline electric field splitting of order $2 \mathrm{eV}$ ). The intersite spin-dependent interactions, expressed as the molecular field acting on the Ni moment, amounts to $500 \mathrm{~T}$.

The spin and orbital moments are parallel - the total moment amounts to $2.53 \mu_{\mathrm{B}}$ at $0 \mathrm{~K}$. With temperature all moments decrease vanishing at the magnetic-ordering temperature. Such large orbital moment, more than $20 \%$ of the total moment, proves that in compounds containing $3 d$ ions the orbital moment has to be "unquenched". We point out that the spin-orbit coupling is fundamentally essential for the existence of this moment. Recent magnetic X-ray experiments of Fernandez et al. have revealed the orbital moment of $0.34 \mu_{\mathrm{B}}$ at room temperature. We are taking these values, 0.54 and $0.34 \mu_{\mathrm{B}}$, to be in nice agreement owing to the fact that the orbital moment becomes larger at low temperatures. In fact, we can say on the basis of our calculations that the spin-orbit coupling is indispensable for the physically-adequate description of magnetic and electronic properties. Obviously the present results call for more advanced solid-state physics theory that will take these facts into account. The need is unavoided as the orbital moment becomes revealed in modern experiments [10]. 
In conclusion, the substantial orbital moment of $0.54 \mu_{\mathrm{B}}$ (at $0 \mathrm{~K}$ ) in $\mathrm{NiO}$ has been found in close agreement with the recent experimental finding. Such large orbital moment, more than $20 \%$ of the total moment of $2.53 \mu_{\mathrm{B}}$, proves that in compounds containing $3 d$ ions the orbital moment has to be "unquenched". It turns out that the spin-orbit coupling is indispensable for description of magnetic and electronic properties of $3 d$-ion compounds.

\section{References}

[1] K. Terakura, A.R. Williams, T. Oguchi, J. Kubler, Phys. Rev. Lett. 52, 1830 (1984).

[2] D.I. Korotin, S.Yu. Ezhov, I.V. Solovyev, V.I. Anisimov, D.I. Khomskii, G.A. Sawatzky, Phys. Rev. B 54, 5309 (1996).

[3] I. Solovyev, N. Hamada, K. Terakura, Phys. Rev. B 53, 7158 (1996).

[4] J. Bała, A.M. Oleś, J. Zaanen, Phys. Rev. Lett. 72, 2600 (1994).

[5] M. Abbate, R. Potze, G.A. Sawatzky, A. Fujimori, Phys. Rev. B 49, 7210 (1994).

[6] R.J. Radwański, presented at European Conf. Physics of Magnetism 93, Poznań 1993, Book of Abstracts, O.3, p. 79.

[7] R.J. Radwański, presented at the VII National Symp. on High-temperature Superconductivity, Międzyzdroje 1997, Book of Abstracts, P2.14.

[8] A. Abragam, B. Bleaney, in: Electron Paramagnetic Resonance of Transition Ions, Clarendon Press, Oxford 1970, p. 372.

[9] R.J. Radwański, J. Phys., Condens. Matter 8, 10467 (1996).

[10] V. Fernandez, C. Vettier, F. de Bergevin, C. Giles, W. Neubeck, Phys. Rev. B 57, 7870 (1998). 